NÚMERO 5 | agosto 2021 - enero 2022 | 126-142

DOI: 10.22201/ffyl.nuevaspoligrafias.2022.5.1554

Revista de teoría literaria y literatura comparada

Recibido: 13-08-2021 | Aceptado: 23-09-2021

\title{
Formato, INC.: REFLEXIONES SOBRE ESTILOS DE CITACIÓN Y ESTUDIOS LITERARIOS ${ }^{1}$
}

\author{
Formatting, InC.: Reflections on Citation STyles And Literary Studies
}

\author{
David Pruneda Sentíes \\ Facultad de Filosofía y Letras \\ Universidad Nacional Autónoma de México | México \\ Contacto: davidpruneda@filos.unam.mx
}

\begin{abstract}
Resumen
El artículo reflexiona acerca de los efectos que han tenido los formatos y estilos de citación en la práctica de los estudios literarios estadounidenses. En primer lugar, reviso de manera panorámica los estilos más utilizados en Estados Unidos, aquéllos respaldados y distribuidos por la University of Chicago Press, por la American Psychological Association (APA) y por la Modern Language Association (MLA). La exitosa inserción de los estilos de citación es una consecuencia de la orientación epistemológica adoptada por las universidades estadounidenses durante la segunda mitad del siglo xix, las cuales emularon el sistema educativo superior alemán. En segundo lugar, reflexiono sobre la relación entre la forma y el contenido del texto académico, puesto que una está íntimamente ligada al otro. Ésta es una discusión muy socorrida cuando se analizan textos literarios, pero no ha sido tan desarrollada en relación con la escritura académica. Finalmente, hago un breve estudio de caso sobre la Modern Language Association para esbozar una propuesta teórica y metodológica que pretenda motivar el estudio de la influencia de los estilos de citación en las dinámicas
\end{abstract}

\begin{abstract}
The paper ponders on the effects of formatting and citation styles in the academic production of American literary studies. Firstly, I revise the most used citation styles in the United States, those enforced and distributed by the University of Chicago Press, the American Psychological Association (APA), and the Modern Language Association (MLA). The successful incorporation of citation styles in academia is a consequence of the epistemological orientation adopted by American universities during the second half of the nineteenth century by following the German university model. Secondly, I reflect on the relationship between form and content in an academic text, as both dimensions are closely intertwined. This is a discussion over which literary studies has agonized tirelessly, but it is often overlooked in the case of academic writing. Finally, I present a brief case study about the Modern Language Association to sketch a theoretical and methodological proposal to study the influence citation styles have had on the dynamics of knowledge production in current academic disciplines. Thus, in this paper I argue that citation
\end{abstract}

1 Algunas ideas contenidas en este artículo son parte de la investigación que realizo para mi tesis de doctorado titulada Lecturas adjetivadas: la protocolización de las prácticas de lectura académica en los estudios literarios estadounidenses (2000-2020). 
de producción de conocimiento de las disciplinas académicas actuales. Es así que en este artículo argumento que los formatos y estilos de citación moldean ideológica y epistemológicamente las investigaciones realizadas en los estudios literarios estadounidenses. Los efectos de los formatos y estilos de citación son consecuencia de los significados explícitos e implícitos que son trasmitidos tanto por las instituciones que los proponen, como por los manuales en los que se materializan sus lineamientos y sugerencias.

Palabras clave: estudios literarios estadounidenses; formato y estilos de citación; APA; Chicago; MLA; ideología de la forma; agencias de la forma styles shape research in American literary studies, both ideologically and epistemologically. The effects of formatting and citation styles are a consequence of explicit and implicit messages transmitted by the associations and the manuals that promote their formatting guidelines and suggestions.

Keywords: American literary studies; formatting and citation styles; APA; Chicago; MLA; ideology of form, agencies of form

$\mathrm{D}$ esde la formalización y profesionalización de la mayoría de las disciplinas universitarias a finales del siglo XIX, la academia estadounidense ha diseñado instituciones e instrumentos que le han permitido impulsar la investigación y la producción de conocimiento. En la actualidad, el uso de un formato y un estilo de citación es una condición sine qua non para socializar el trabajo académico, por lo que no es posible menospreciar el papel que juegan estas convenciones en el circuito editorial y en la práctica de quienes se dedican a la investigación. El propósito de este artículo es identificar algunos de los efectos de los formatos y estilos de citación en los estudios literarios en Estados Unidos. Argumento que, desde que su uso se ha vuelto generalizado en los departamentos de literatura, los formatos y estilos de citación han moldeado ideológica y epistemológicamente la producción escrita de los estudios literarios estadounidenses. Estos efectos son consecuencia de los significados explícitos e implícitos que son trasmitidos tanto por las instituciones que proponen los formatos y estilos de citación, como por los manuales en los que se materializan sus lineamientos y sugerencias.

Con el afán de demostrar lo anterior, primero hago una revisión panorámica de los principales formatos y estilos de citación utilizados en la academia en Estados 
Unidos. En segundo lugar, exploro algunas de las reflexiones en torno a la relación entre la forma y el contenido de un texto, dado que, si bien ha sido intensamente analizada en el caso del texto literario, poco se ha discutido con respecto al texto académico. Asimismo, la relación entre forma y contenido es el punto de partida para el esclarecimiento de la influencia epistemológica e ideológica de los formatos y estilos de citación en las disciplinas universitarias. Finalmente, llevo a cabo un breve estudio de caso sobre el formato y estilo de citación de la Modern Language Association.

\section{Style Galore}

Un formato y un estilo de citación comprenden un conjunto de lineamientos formales que determinan la manera en la que se presenta la información relativa a las fuentes documentales que respaldan tanto la información fáctica como los argumentos contenidos en un texto académico. Estos objetos se han vuelto prácticamente omnipresentes en la labor académica en Estados Unidos. Si bien esta situación es el resultado de la combinación de múltiples factores de diversa naturaleza (pedagógica, institucional, política, económica), es razonable decir que el éxito de los formatos y estilos de citación radica en su consonancia con una manera particular de entender la función del trabajo realizado dentro de las universidades.

De acuerdo con Gerald Graff y Michael Warner (1989: 5-9), en las últimas tres décadas del siglo XIx, las instituciones de educación superior en Estados Unidos decidieron adoptar un modelo semejante al de las universidades alemanas de la época, en donde había programas de posgrado y se privilegiaba la investigación por encima de la educación. Este cambio de rumbo no sólo fue consecuencia de los intereses particulares de los académicos estadounidenses que regresaron de Europa con estudios de posgrado -inexistentes hasta ese entonces en su tierra natal- y que quedaron maravillados por la figura del profesor universitario en Alemania, ${ }^{2}$ sino que también fue parte de una transformación social más amplia que fue impulsada por procesos

2 En German Universities: A Narrative of Personal Experience (1874), James Morgan Hart presenta en gran estima las dinámicas institucionales de la universidad alemana. Sobre el papel del profesor universitario, Hart (1989) señala que el profesor universitario no es un maestro, sino un especialista y por lo tanto su responsabilidad no recae en el éxito de sus estudiantes, sino en la calidad de su propia instrucción: "No man can become a professor in a German university without having given evidence, in one way or another, that he has pursued a certain line of study, and produced results worth to be called novel and important. In other words, to become a professor, he must first have been a special investigator" (22). 
de industrialización cada vez mejor consolidados. Como se mencionó al inicio de este artículo, en Estados Unidos ésta fue la época en la que diversas actividades laborales se profesionalizaron, como la abogacía, la medicina, las ciencias sociales y, por supuesto, los estudios literarios (Graff y Warner, 1989: 11).

Lo anterior es relevante para explicar el éxito de los formatos y estilos de citación en la práctica académica porque toda profesionalización implica el diseño de formas de organización. Esto ocasionó la aparición de múltiples asociaciones, clubes y fundaciones que revelan el impulso por organizar la sociedad en segmentos dedicados a actividades específicas. Según William R. Parker (1953), durante la segunda década del siglo xIx "Organization was in the air" (27). ${ }^{3}$ Estas asociaciones apuntalaron la nueva dirección de las universidades hacia la investigación porque facilitaron la comunicación entre profesores que comenzaron a pensarse como especialistas cuya principal labor era más bien la producción de conocimiento. Una de las maneras de promover esta comunicación fue la creación de formatos y estilos de citación. No es una sorpresa, por lo tanto, que los tres formatos y estilos más utilizados en la academia estadounidense sean aquéllos diseñados y distribuidos por asociaciones de este tipo: la University of Chicago Press (Chicago), la American Psychological Association (APA) y la Modern Language Association (MLA).

El primer estilo de citación que apareció en la escena editorial académica de Estados Unidos fue creado por la University of Chicago Press y respondió a la necesidad de estandarizar la manera en la que los tipógrafos convertían manuscritos en textos impresos. El breve documento elaborado por los trabajadores de la editorial se distribuyó en forma de panfleto entre la comunidad universitaria de Chicago y en 1906 se convirtió en un libro: el Manual of Style: Being a Compilation of the Typographical Rules in Force at the University of Chicago Press, to Which Are Appended Specimens of

3 Parker(1953) enlista algunos de los grupos que surgieron en Estados Unidos durante el siglo xIx: "The British Philological Society (1842) was about to publish the first parts of its great Dictionary (from 1884). The Early English Text Society (1864) was making available rare materials for literary and linguistic research. Suffice it merely to mention the Royal Society of Literature (1823), the Roxburghe (1812) and Hunterian (1873) clubs, the Browning, Camden, Chaucer, Harleian, Percy, Spenser, Shakespeare and New Shakespeare societies, all of nineteenth-century origin. In the United States this same period had seen the birth of the American Antiquarian (1812), Oriental (1842), and Numismatic (1858) societies, the Society of Biblical Literature and Exegesis (1880), the National Education (1857), American Philological (1869), and American Library (1876) associations, the American Associations for the Advancement of Science (1848) and of University Women (1882), and the Archaeological Institute of America (1879). The year after the MLA was founded, the historians organized their national society. In the following year, it was the economists" (27). 
Types in Use (The University of Chicago Press, 2017). Es importante destacar que este primer manual se presenta como "the embodiment of traditions, the crystallization of usages, the blended product of the reflections of many minds" (The University of Chicago Press, 1906: v), lo que evidencia tanto su carácter colectivo como su interés en difundir una cierta manera de hacer las cosas. Esta manera se describe de forma peculiar con los adjetivos y los adverbios que caracterizan el conjunto de reglas formales que sugiere el documento: "As it stands, this Manual is believed to contain a fairly comprehensive, reasonably harmonious, and wholesomely practical set of work-rules for the aid of those whose duties bring them into direct contact with the Manufacturing Deparment of The Press" (The University of Chicago Press, 1906: vII). Es claro que la retórica del texto pretende convencer a quien lo lee del raciocinio detrás de su elaboración y de su utilidad en la producción editorial.

En la actualidad, el estilo Chicago es normativo principalmente en la investigación en economía, en historia y en las artes. A su vez, el Chicago Manual of Style se encuentra en su decimoséptima edición y se piensa como "the authoritative reference work for authors, editors, proofreaders, indexers, copywriters, designers, and publishers" (The University of Chicago Press, 2017: s. p.). Esta imagen propia es recurrente en otros manuales de estilo de citación, lo que sin duda podría definirse como un rasgo idiosincrático de este tipo de documentos y que, como se ve más adelante en este artículo, tiene la capacidad de modificar ideológicamente las prácticas académicas de las disciplinas universitarias.

El estilo APA se utiliza primordialmente en los campos de las ciencias naturales y sociales, la educación y la psicología. De acuerdo con su página de internet, el estilo surgió en 1929, "when a group of psychologists, anthropologists, and business managers convened and sought to establish a simple set of procedures, or style guidelines, that would codify the many components of scientific writing to increase the ease of reading comprehension" (American Psychological Association, 2019: s. p.). De esta cita merece la pena subrayar tanto el tono con el que se presenta la génesis del estilo APA, como su propósito explícito. Por un lado, en una retórica similar a la del estilo Chicago, la aseveración de que este estilo se haya concebido como un conjunto "simple" de procedimientos y lineamientos estilísticos acarrea un bagaje ideológico que no puede pasarse por alto, dado que calificar algo de simple o sencillo supone no sólo su fácil entendimiento, sino también su fácil aceptación y asimilación. Este tipo de discurso tiende a sustentarse en el sentido común hegemónico; es decir, los lineamientos formales incluidos en la sección del Psychological Bulletin de 1929 
titulada "Instructions in regard to preparation of manuscript", además de ser lógicos y evidentes, son del interés de todas las personas involucradas en su uso.

Por el otro lado, la intención de incrementar la comprensión de lectura de la escritura científica avanza sobre una línea similar, puesto que toma como punto de partida una manera específica de entender este tipo de textos y, aun más importante, parte de la capacidad de emitir un juicio en relación con su grado de inteligibilidad:

When style works best, ideas flow logically, sources are credited appropriately, and papers are organized predictably and consistently. People are described using language that affirms their worth and dignity. Authors plan for ethical compliance and report critical details of their research protocol to allow readers to evaluate findings and other researchers to potentially replicate the studies. (American Psychological Association, 2019: s. p.)

Llama la atención la rapidez con la que se asocian cuestiones como el valor, la dignidad y la ética de las personas con el uso de un estilo de citación. En ese sentido, resulta difícil no identificar un potente mensaje ideológico que se transmite explícitamente en la página de internet del estilo APA, pero también se difunde implícitamente a través de los lineamientos formales contenidos en sus manuales.

En cuanto al surgimiento del estilo MLA, por iniciativa de Carleton Brown y Cyril Arthur Peerenboom, se desarrolló en 1931 la "Style Sheet", en donde se especificaron algunas directrices editoriales para los manuscritos que fueran enviados a la $P M L A .{ }^{4}$ La propuesta fue aprobada por el editor de la revista, Percy W. Long, quien afirmó que "The immediately pressing problem of the editor is to secure within reason a uniform style in such little things as quotations, footnotes, and bibliographical references" (citado en Modern Language Association, 2021a: s. p.). La primera "Style Sheet" consistió únicamente de tres páginas incluidas en la PMLA y, además de los lineamientos señalados por Long, propuso una estandarización en la puntuación y la ortografía.

Las tres páginas de la $P M L A$ se convirtieron en un pequeño manual que se publicó por separado en 1951: "Derived from the PMLA style sheet, this thirty-onepage, stapled booklet was the association's first published style guide [...] In addition

4 PMLA son las siglas de la revista oficial de la MLA desde 1889, Publications of the Modern Language Association. En ésta, la asociación difunde información relativa a sus actividades: publicaciones, convenciones, reportes administrativos, etcétera. 
to providing guidance on how to cite sources, it offered brief sections on manuscript preparation, punctuation, spelling, and other style matters" (Modern Language Association, 2021a: s. p.). Después de su segunda edición en 1970, este panfleto fue sustituido en 1977 por la primera edición del MLA Handbook for Writers of Research Papers, Theses, and Dissertations, que, a diferencia de su predecesor, ha sobrevivido por varias ediciones. ${ }^{5}$ De éstas merece la pena destacar la sexta, de 2003, en donde se modificó el nombre de la publicación a MLA Handbook for Writers of Research Papers, y la octava, de 2016, en la que nuevamente se reformó el nombre al lacónico MLA Handbook.

En el tercer apartado de este artículo profundizo en las características normativas y en los mensajes ideológicos transmitidos por el estilo MLA. Por lo pronto, es pertinente apuntar que el uso de los formatos y estilos de citación en las publicaciones académicas se encuentra en consonancia con la orientación hacia la investigación elegida por las universidades estadounidenses a partir de su adopción del modelo institucional alemán. Por lo tanto, es razonable afirmar que la academia en Estados Unidos ha encontrado en los formatos y estilos de citación una forma de organización y comunicación eficientes que han favorecido la producción de conocimiento. El siguiente apartado de este artículo sienta una base teórica para comenzar a identificar los efectos epistemológicos e ideológicos de este fenómeno.

\section{Las agencias de la forma}

No cabe duda de que la relación entra la forma y el contenido de un texto es uno de los temas de reflexión cardinales en los estudios literarios. De acuerdo con Terry Eagleton (2010), "La mayoría de los críticos siempre insiste en que estos dos aspectos de una obra son inseparables. De hecho, esta doctrina está tan arraigada en los críticos literarios como lo estaba para la Inquisición la existencia de brujas" (81). La

5 Las ediciones del Handbook han sido más o menos constantes a lo largo de los años: segunda: 1984; tercera: 1988; cuarta: 1995; quinta: 1999; sexta: 2003; séptima: 2009; octava: 2016; y novena: 2021. Además, vale la pena mencionar que, paralelamente, la MLA publicó el MLA Style Manual and Guide to Scholarly Publishing por primera vez en 1985, con la intención de complementar el Handbook, pues a diferencia de éste, que estaba dedicado a alumnos y profesores en nivel medio-superior y superior, el Manual estaba dirigido a estudiantes de posgrado, académicos, profesores universitarios, escritores profesionales y editores. Esta publicación fue descontinuada en 2016, cuando la MLA declaró que la octava edición del Handbook sería la única autoridad en el estilo de la asociación. 
longevidad de esta discusión da cuenta de su complejidad porque puede ser abordada desde diversas perspectivas. Es verdad que no es posible separar el significado de un mensaje de la manera en la que se transmite, pero también es cierto que la distinción entre forma y contenido es discernible tanto en lo concreto como en lo abstracto; inclusive, "el mero hecho de que usemos dos términos diferentes para ellos ya indica que no son idénticos" (Eagleton, 2010: 81). Es de mi parecer que la postura más productiva para trabajar con este fenómeno es concebirlo como una relación dinámica en la que ambos componentes se moldean mutuamente en diferentes grados y dependiendo de los momentos específicos del texto en los que se observa esta relación.

Un desarrollo teórico que no necesariamente comulga con el carácter relativizador de la aseveración anterior, pero que resulta interesante como un punto de partida para conducir la discusión sobre la forma y el contenido en relación con los textos académicos, es la propuesta de lectura de Fredric Jameson en The Political Unconscious (1979). El propósito de esta práctica interpretativa es explicar por qué los objetos culturales - como una máscara tribal o un texto literario- tienen la forma que tienen. Sustentada principalmente por las teorías marxista y psicoanalítica, la hipótesis de Jameson es que las características formales de un objeto cultural son soluciones simbólicas a contradicciones sociales que no pueden resolverse en la realidad, por lo que la tensión que éstas provocan en las sociedades encuentra un alivio en la producción cultural. Jameson (1983) parte del supuesto de que el texto reprime un contenido ideológico que ha permanecido "unrealized in the surface of the text, [and] which have failed to become manifest in the logic of the narrative" (33). De acuerdo con esta postura, en su ejercicio de lectura el crítico se dedica a diagnosticar este contenido subterráneo a partir de síntomas que identifica en la forma del texto, cuyos elementos se interpretan como las soluciones simbólicas a las contradicciones sociales irresolubles.

Como escribí al inicio del párrafo anterior, la propuesta de Jameson para el estudio de la relación entre forma y contenido no se mueve sobre la misma línea que este artículo, debido a que mientras que Jameson aborda este fenómeno a partir de una postura totalizante - es decir, supone que es posible diseñar una interpretación que explique de manera sintética la relación entre todos los aspectos formales y semánticos de una obra-, aquí se asume la postura de que esta relación es diferente en distintos momentos de un mismo texto. No obstante, es relevante comentar que la perspectiva totalizante que adopta Jameson le permite plantear el concepto de "ideología de la forma", que se refiere al mensaje ideológico que es transmitido no por el 
contenido semántico de una obra, sino por sus características y procesos formales. Mediante el estudio de la ideología de la forma, "it has become possible to grasp such formal processes as sedimented content in their own right, as carrying ideological messages of their own, distinct from the ostensible or manifest content of the works" (Jameson, 1983: 84). Dado que un estilo de citación se compone de un conjunto de características formales que interactúan con un contenido semántico, la noción de la ideología de la forma resulta de utilidad para pensar no sólo en el mensaje ideológico implícito que transmiten, sino también en la manera en la que éste moldea las ideas de un texto académico.

La propuesta de Jameson abre la puerta a la reflexión sobre el efecto de las formas textuales en el ámbito social extratextual. Éste es el camino que han decidido seguir algunos de los análisis neoformalistas, específicamente el trabajo de Caroline Levine (2015), quien se pregunta si la distinción entre lo formal y lo social es realmente pertinente. A partir de una definición amplia de forma como "an arragement of elements - an ordering, patterning, or shaping" (3), Levine (2015) propone que los estudios literarios incluyan en sus análisis patrones de experiencia sociopolítica, dado que "politics involves activities of ordering, patterning, and shaping. And if the political is a matter of imposing and enforcing boundaries, temporal patterns, and hierarchies on experience, then there is no politics without form" (3). A diferencia de ciertos análisis formalistas, como los del New Criticism, que limitaban su labor al estudio pormenorizado de la función intratextual de las formas en un texto literario - poniendo en suspenso la dimensión extratextual de las obras-, el neoformalismo de Levine (2015) se interesa por el impacto de las formas textuales en la realidad: "Forms matter, in these accounts, because they shape what it is posible to think, say, and do in a given context" (5). En otras palabras, además de hablar de una ideología de la forma, es importante ponderar las agencias de la forma, que pueden entenderse como los efectos que tienen los arreglos formales de un texto en la vida y las prácticas sociales de las personas. ${ }^{6}$

Tanto Jameson como Levine trabajan sus ideas teóricas a partir de análisis de textos literarios, lo que dirige su atención hacia prácticas sociales muy diversas; es decir, ellos observan cómo lo económico (Jameson) y lo político (Levine) configuran

6 Levine (2015: 6-11) llama affordance a la capacidad de las formas textuales de tener una consecuencia en las prácticas sociales. Sin duda, las agencias de la forma es una noción que se traslapa con la affordance ofform de Levine. 
y son configurados por formas textuales. Puesto que en este artículo el interés radica en textos académicos, es necesario tomar una dirección paralela que especialmente se concentre en lo epistemológico, dado que, en cuanto que disciplina académica orientada hacia la investigación, los estudios literarios estadounidenses tienen como uno de sus propósitos la producción de conocimiento en torno a la literatura. En otras palabras, cuando se estudian las formas en el texto académico desde la perspectiva de las agencias de la forma, la atención no está puesta en cómo la gente trabaja o se organiza políticamente, sino en cómo lee.

En este punto es pertinente decir que las prácticas de lectura académica representan casos específicos de actos de recepción textual, por lo que su estudio debe distinguirse - aunque no separarse por completo- del trabajo de la estética de la recepción literaria y de las teorías de la respuesta del lector. Ya sea que se explore el acto de lectura de manera abstracta o concreta, los estudios de recepción literaria se interesan por explicar el fenómeno en el que una persona entra en contacto con un texto a partir de espacios de indeterminación y de horizontes de expectativas, lo que resulta en la producción de significados compuestos tanto por el material que aporta el texto, como por el que aporta quien lee. En cambio, el estudio del texto académico debe dar por sentado este proceso y concentrar su energía en explicar el fenómeno en el que una persona da cuenta de un acto de lectura.

Éste, por supuesto, no es aleatorio ni espontáneo, sino que debe cumplir con una serie de lineamientos que provienen de diversos orígenes, entre los que resalta la escritura académica misma. Para Elaine Auyoung (2020), "when we refer to what literary critics do as reading, we obscure how much their interpretations are shaped by unspoken conventions involved in writing literary criticism" (94). Resulta razonable aseverar, por lo tanto, que la manera en la que se escribe en los estudios literarios estadounidenses influye en las lecturas de las que los textos académicos dan cuenta. De esta manera, las formas textuales, en general, y los formatos y estilos de citación, en particular, moldean las prácticas de lectura académica, que son las principales herramientas epistemológicas de los estudios literarios.

Así como Jameson plantea que el estudio de la ideología de la forma en los textos literarios permite pensar los procesos formales como cargadores de significados ideológicos, el estudio de las agencias de la forma en los textos académicos — partiendo de la premisa de que la relación entre forma y contenido es dinámica y de influencia mutua - revelará que hay algunos temas y enfoques en las investigaciones de los estudios literarios que son fomentados y otros que son inhibidos por procesos formales 
y por estilos de citación. En el tercer apartado de este artículo se desarrolla un breve estudio de caso para esbozar algunas de las agencias de la forma del estilo MLA en la producción escrita de los estudios literarios estadounidenses.

\section{Una "Biblia del estilo"”}

Para entender la influencia del estilo MLA en los estudios literarios, es necesario hacer una breve revisión de la historia de la asociación, puesto que su devenir arroja luz sobre la naturaleza del contenido ideológico que transmite en sus publicaciones y, específicamente, en el MLA Handbook, cuyo uso se ha vuelto muy significativo en la producción de las investigaciones en humanidades. Por supuesto, esta situación es el resultado de una serie de factores que han colocado a esta institución como, de acuerdo con su sitio de Internet, una de las más grandes asociaciones académicas del mundo, con más de 24000 miembros en 100 países (Modern Language Association, 2021b).

La MLA de hoy en día se fundó en 1883 tras su primera convención, pero fue en la segunda convención de 1884 en donde Alonzo Williams, profesor de Brown University, leyó seis declaraciones sobre las que se basaría la primera constitución de la asociación. ${ }^{8}$ A partir de la tercera de estas declaraciones evolucionó la misión actual de la MLA:

7 En la contraportada de la sexta edición del MLA Handbook for Writers of Research Papers (2003), Newsweek se refiere a la publicación como "[t]he style bible for most college students" (Modern Language Association, 2021a). En su reseña sobre la tercera edición del MLA Style Manual and Guide to Scholarly Publishing, James M. Clawson (2009) lo nombra "this Bible of the humanities" (235). A su vez, en el prólogo de la octava edición del MLA Handbook, Rosemary G. Feal, la directora ejecutiva de la asociación, lo llama "the 'style bible' for generations of students" (Modern Language Association, 2016: s. p.).

8 Las seis declaraciones son las siguientes: "I. The name of this Society shall be "The Modern Language Association of America"; "II. Any person approved by the Executive Council may become a member by the payment of three dollars, and may continue a member by the payment of the same amount each year"; "III. The object of this Association shall be the advancement of the study of the Modern Languages and their Literatures"; "IV. The officers of the Association shall be a President, Secretary, Treasurer, and nine members, who shall together constitute the Executive Council, and these shall be elected annually by the Association"; "V. The Executive Council shall have charge of the general interests of the Association, such as, the election of members, calling of meetings, selection of papers to be read, and the determination of what papers shall be published"; "VI. The Constitution may be amended by two-thirds vote at any annual meeting, provided the proposed amendment has received the approval of the Executive Council" (Cook, 1884: XIX). 
The Modern Language Association promotes the study and teaching of languages and literatures through its programs, publications, annual convention, and advocacy work. The MLA exists to support the intellectual and professional lives of its members; it provides opportunities for members to share their scholarly work and teaching experiences with colleagues, discuss trends in the academy, and advocate humanities education and workplace equity. The association aims to advance the many areas of the humanities in which its members currently work, including literature, language, writing studies, screen arts, digital humanities, pedagogy, and library studies. The MLA facilitates scholarly inquiry in and across periods, geographic sites, genres, languages, and disciplines in higher education that focus on communication, aesthetic production and reception, translation, and interpretation. Through the Association of Departments of English and the Association of Departments of Foreign Languages, the MLA also supports the work of department chairs and directors of graduate studies. (Modern Language Association, 2021b: s. p.)

Más allá de los propósitos de promoción de las humanidades y de apoyo a sus miembros, lo que más me interesa en este artículo es la última parte de la cita anterior, en donde la MLA se presenta a sí misma como una facilitadora de la difusión académica a través del espacio, del tiempo y de las lenguas.

La imagen propia de la MLA es relevante puesto que proyecta sus intenciones hegemónicas al regular las condiciones espaciales, temporales y lingüísticas en las que se realiza la producción de escritura académica en Estados Unidos. Ian Baucom (2001) articula una analogía entre la MLA y la corporación de las compañías de las Indias. Más que una forma de organización y de administración de bienes, las compañías de las Indias se han vuelto una figura conceptual que en la actualidad permite pensar en las formas que el capital adopta en un momento de globalización, lo cual se debe a que gracias a esta corporación emergió la posibilidad de la forma global: "What this concept of global form reveals is that globalization must be apprehended not simply as a process of enlargement or expansion but also, and perhaps more important, as one of contraction and concentration" (Baucom, 2001: 160). Las compañías de las Indias permitieron a los imperios conectar económicamente sus ciudades capitales con los extremos más alejados de sus territorios de ultramar, es decir, expansión; además, facilitaron establecer un intercambio en el que diversos espacios, diversos tiempos y diversos recursos se condensaron en un solo espacio, un solo tiempo y una sola moneda, es decir, concentración (Baucom, 2001: 160). Baucom argumenta 
que el desarrollo histórico de la MLA presenta correspondencias perturbadoras con los procesos de las compañías de las Indias, en cuanto que se ha vuelto uno de los principales centros de intercambio de capital intelectual en el mundo.

Quizás uno de los fenómenos más llamativos de este proceso se encuentra cifrado en el nombre de la asociación porque

it is not simply the MLA, it is the MLAA, the Modern Language Association of America. With that final recognition in place (the recognition of the way in which, through the most casual of citational practices, we tend at once to globalize the MLA implicitly and to name America as the site of the global by not speaking its name), the speculative fantasy of this project begins to look all too familiar... (Baucom, 2001: 169)

Familiar no sólo porque este proceso asemeja el de las compañías de las Indias, sino también porque es necesario recordar el destino del nombre del Handbook, que presenta correspondencias inquietantes: primero, MLA Handbook for Writers of Research Papers, Theses, and Dissertations (1977); después, MLA Handbook for Writers of Research Papers (2003); y ahora, MLA Handbook (2016). El fenómeno de concentración del que habla Baucom se vuelve tangible.

Los efectos ideológicos de las agencias de la forma del estilo MLA ya pueden intuirse en función de lo que he detallado arriba, pero merece la pena puntualizar tres de ellos relacionados con los impulsos expansionistas evidentes en el devenir de la asociación. En primer lugar, el estilo MLA habilita un espacio para llevar a cabo una discusión en un marco de normas establecidas que pueden seguirse independientemente del origen geográfico de los textos que se estudien. De manera paradójica, el estilo establece una serie de fronteras formales con el objetivo de que las fronteras geográficas no representen un obstáculo, sino una oportunidad más. Esto ha fomentado en los departamentos de literatura estadounidenses tanto la exploración de literaturas provenientes de diferentes lugares, como la participación de académicos de distintos orígenes y en diferentes partes del mundo. En segundo lugar, el estilo habilita una contemporaneidad en donde las voces del ahora pueden dialogar con las del ayer; es decir, cuando un texto académico incluye una cita, su idea se vuelve contemporánea a medida que es revitalizada por el discurso principal. De esta manera, se revela el carácter heterocrónico del texto académico, puesto que las comillas utilizadas para señalar la cita funcionan como una membrana visible que separa y une el 
presente con el pasado. Además, el estilo MLA es un documento vivo que, en cuanto que se modifica y se edita con relativa frecuencia, también se proyecta a un futuro. En tercer lugar, hay que señalar el efecto que tienen las agencias de la forma en aspectos lingüísticos, pues el estilo MLA permite que diferentes lenguas convivan en un mismo texto. Por ejemplo, las notas al pie habilitan la posibilidad de incluir traducciones, de manera que las citas de los textos que son estudiados pueden presentarse en el idioma original sin que esto represente un problema para la comprensión del texto académico. No obstante, es importante recordar que el circuito editorial académico en Estados Unidos se conduce principalmente en inglés, por lo que cualquier contribución que pretenda ser publicada debe estar escrita en esta lengua. De esta manera, el texto académico podría verse como un mapa de irregularidades espaciales, temporales y lingüísticas, que gracias a las agencias de la forma del estilo de citación reúne en un "aquî", en un "ahora" y en "asî" las diferentes voces de otros tiempos y de otros lugares. Lo anterior nuevamente da cuenta del proceso de concentración del que habla Baucom, puesto que por medio del formato y estilo de citación de la MLA, la academia estadounidense ha concentrado una gran cantidad de capital intelectual proveniente de diversas partes del mundo.

Los efectos epistemológicos de las agencias de la forma del estilo MLA pueden dividirse en aquéllos que se relacionan con la escritura y en aquéllos que se relacionan con la lectura; es decir, para identificar la influencia epistemológica del estilo MLA en los estudios literarios estadounidenses es necesario centrar la atención en las estrategias metodológicas que utilizan para la producción de conocimiento. El efecto de las agencias de la forma en la escritura es relativamente evidente: basta con echar un vistazo a la manera en la que se ha moldeado la manera de escribir en función de las recomendaciones y los lineamientos del estilo MLA. De hecho, una de las dos partes en las que está dividida la octava edición del MLA Handbook está dedicada a "The Mechanics of Scholarly Prose".

En cuanto a la lectura, es pertinente puntualizar que la práctica actual del estudio literario involucra la revisión y el análisis no sólo de la obra literaria, sino también de un conjunto de textos críticos y teóricos que respaldan los argumentos de una interpretación. Por un lado, la otra parte de la octava edición del MLA Handbook está dedicada al proceso de investigación, de manera que esta sección contiene subtítulos como "Think: Evaluating Sources", "Select: Gathering Information about Your Sources" y "Organize: Creating Your Documentation". Por otro lado, es relevante mencionar también la importancia que este estilo da al nombre de los autores y las autoras, tanto 
en el formato general (el nombre de quien escribe el texto debe aparecer en la esquina superior izquierda de la primera página y en la esquina superior derecha junto al número de cada página), como en la manera de referir las fuentes parafraseadas o citadas. El impacto que esto tiene en la lectura llevada a cabo para la producción de un texto académico puede ser variable, pero es razonable suponer que esto motiva a que la búsqueda de información se lleve a cabo en función de autores y autoras y no necesariamente en función de fechas o temas.

Finalmente, no está de más apuntar que buena parte del impacto que ha tenido el formato y el estilo MLA en la práctica de los estudios literarios es una consecuencia de su inserción en el sistema universitario en Estados Unidos. Éste es un proceso que inició formalmente en 1951, cuando la MLA Style Sheet se distribuyó entre investigadores y estudiantes de posgrado. En la página legal de este documento se afirma que no tiene sentido instruir a futuros académicos en una forma para preparar artículos y tesis, si las editoriales y las revistas en las que publicarán sus trabajos requieren de un formato distinto (Modern Language Association, 2021a). En otras palabras, los estudiantes universitarios comenzaron no sólo a recibir los contenidos conceptuales de las materias, sino también los lineamientos del formato y estilo MLA desde mediados del siglo pasado y continúan haciéndolo en la actualidad.

\section{Conclusión}

Lo que mal que bien se ha esbozado en este artículo es apenas el tanteo de algunas intuiciones en torno al estudio del texto académico en cuanto que género discursivo. Consideraciones de mayor calado requerirían de cavilaciones más profundas y mejor documentadas. Hace falta, para empezar, preguntarse con más detalle cuál es la influencia de cada elemento de los formatos y los estilos de citación en el contenido del texto académico, qué tipo de análisis se motivan y qué tipo de análisis se inhiben en la práctica. Inclusive, si se parte del supuesto de que el pensamiento depende de los marcos de referencia que tiene, merecería la pena dar un paso hacia atrás y explorar la injerencia del formato y el estilo de citación en la gestación de las ideas, desde aquéllas que se materializan en ensayos breves, hasta aquéllas que fundamentan tesis doctorales. Además, siguiendo a Baucom (2001) cuando dice que "genres not only emerge from specific historical situations but carry that ideology in themselves as a ghostly aftereffect" (163), debería considerarse que el texto académico porta, apa- 
rentemente sin tapujos, las marcas del poder intelectual; en sus citas, sus paráfrasis, sus referencias y en sus figuras retóricas se deja ver la hegemonía de ciertas ideas y de ciertos autores y autoras. Desde este punto de vista, el texto académico se vuelve una fotografía en alta definición de procesos de dominación cultural. Por último, también sería necesario apuntar a un nivel de problematización superior en el que se busque discutir los formatos y estilos de citación en su calidad de meta-forma textual, pues pueden pensarse como formas que contienen otras formas.

\section{Referencias bibliográficas}

American Psychological Association. (2019). “About APA Style” (en línea). APA Style. Recuperado el 7 de agosto de 2021 de https://apastyle.apa.org/about-apa-style

Auyoung, Elaine. (2020). "What We Mean by Reading." New Literary History, 51(1), 93114. https://doi.org/10.1353/nlh.2020.0004

Baucom, Ian. (2001). "Globalit, Inc.; Or, the Cultural Logic of Global Literary Studies". PMLA, 116(1), 158-72.

Clawson, James M. (2009). "Modern Language Association of America. 2008. The MLA Style Manual and Guide to Scholarly Publishing. 3rd ed. New York: Modern Language Association. $\$ 32.50$ hc. xxiv + 336 pp." [Reseña de The MLA Style Manual and Guide to Scholarly Publishing]. College Literature, 36(3), 234-237. https://doi. org/10.1353/lit.0.0067

Cook, William. (1884). Modern Language Association of America. Proceedings (1), Proceedings at New York, 29, 30, diciembre, XV-Xx.

EAgLETon, Terry. (2010 [2007]). Cómo leer un poema (Mario Jurado, Trad.). Akal.

GrafF, Gerald; WARnER, Michael (Eds.). (1989). The Origins of Literary Studies in America: A Documentary Anthology. Routledge.

Hart, James Morgan. (1989). "From German Universities: A Narrative of Personal Experience". En Gerald Graff y Michael Warner (Eds.), The Origins of Literary Studies in America: A Documentary Anthology. Routledge.

Jameson, Fredric. (1983 [1979]). The Political Unconscious: Narrative as a Socially Symbolic Act. Routledge. 
Levine, Caroline. (2015). Forms: Whole, Rhythm, Hierarchy, Network. Princeton University Press.

Modern Language Association. (2021a). “The History of MLA Style, 1931-2016” (línea de tiempo interactiva, en línea). Modern Language Association. Recuperado el 8 de Agosto de 2021 de https://www.mla.org/About-Us/About-the-MLA/MLAArchives/Time-Lines/MLA-Style

Modern Language Association. (2021b). “MLA’s Mission” (en línea). Modern Language Association. Recuperado el 11 de agosto de 2021 de https://www.mla.org/AboutUs/About-the-MLA/The-MLA-s-Mission

Modern Language Association. (2016). MLA Handbook (8va Edición). Modern Language Association.

PArker, William R. (1953). “The MLA, 1883-1953”. PMLA, 68(4), 3-39. HTtps://Doi. ORG/10.2307/2698983

The University of Chicago Press. (1906). Manual of Style: Being a compilation of the typographical Rules in force at the University of Chicago Press, to which are appended specimens of types in use. The University of Chicago Press.

The University of Chicago Press. (2017). "About The Chicago Manual of Style" (en línea). The Chicago Manual of Style Online. Recuperado el 7 de agosto de 2021 de https://www.chicagomanualofstyle.org/help-tools/about.html

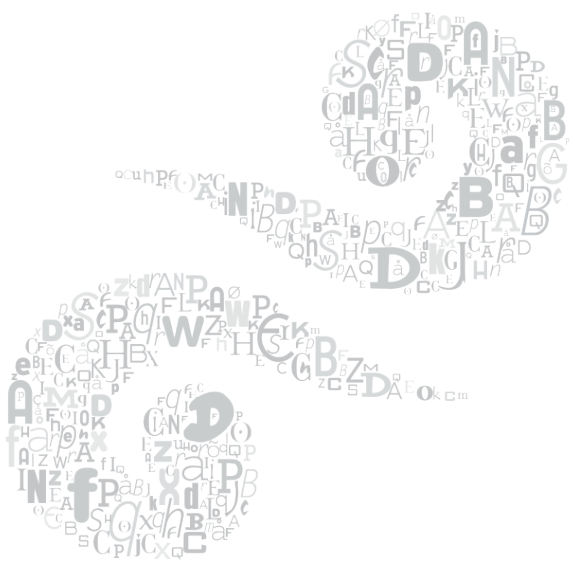

